\title{
BMJ Open Epidemiological impact of achieving UNAIDS 90-90-90 targets for HIV care in India: a modelling study
}

\author{
Manoj V Maddali, ${ }^{1}$ Amita Gupta, ${ }^{1,2}$ Maunank Shah ${ }^{1}$
}

To cite: Maddali MV,

Gupta A, Shah M.

Epidemiological impact of achieving UNAIDS 90-90-90 targets for HIV care in India: a modelling study. BMJ Open 2016;6:e011914.

doi:10.1136/bmjopen-2016011914

- Prepublication history for this paper is available online. To view these files please visit the journal online (http://dx.doi.org/10.1136/ bmjopen-2016-011914).

Received 15 March 2016 Revised 7 June 2016 Accepted 14 June 2016

CrossMark

\footnotetext{
${ }^{1}$ Division of Infectious Diseases, Department of Medicine, School of Medicine, Johns Hopkins University, Baltimore, Maryland, USA

${ }^{2}$ Department of International Health, Bloomberg School of Public Health, Johns Hopkins University, Baltimore, Maryland, USA
}

Correspondence to Manoj V Maddali; manoj.maddali@jhmi.edu

\section{ABSTRACT}

Objective: Recent UNAIDS '90-90-90' targets propose that to end the HIV epidemic by $2030,90 \%$ of persons living with HIV (PLWH) worldwide should know their diagnosis, $90 \%$ of diagnosed PLWH should be on antiretroviral therapy (ART) and $90 \%$ of PLWH on ART should be virally suppressed by 2020 . We sought to quantify the epidemiological impact of achieving these targets in India.

Methods: We constructed a dynamic-transmission model of the Indian HIV epidemic to project HIV infections and AIDS-related deaths that would occur in India over 15 years. We considered several scenarios: continuation of current care engagement (with early ART initiation), achieving 90-90-90 targets on time and delaying achievement by 5 or 10 years.

Results: In the base case, assuming continuation of current care engagement, we project 794000 (95\% uncertainty range (UR) 571 000-1 104 000) HIV infections and 689000 (95\% UR 468 000-976 000) AIDS-related deaths in India over 15 years. In this scenario, nearly half of PLWH diagnosed would fail to achieve viral suppression by 2030. With achievement of 90-90-90 targets, India could avert $392000(95 \%$ UR $248000-559000$ ) transmissions (48\% reduction) and 414000 (95\% UR $260000-598$ 000) AIDS-related deaths (59\% reduction) compared to the base-case scenario. Furthermore, fewer than 20000 (95\% UR 12 000-30 000) HIV infections would occur in 2030. Delaying achievement of targets resulted in a similar reduction in HIV incidence by 2030 but at the cost of excess overall infections and mortality.

Conclusions: India can halve the epidemiological burden of HIV over 15 years with achievement of the UNAIDS 90-90-90 targets. Reaching the targets on time will require comprehensive healthcare strengthening, especially in early diagnosis and treatment, expanded access to second-line and thirdline ART and long-term retention in care.

\section{INTRODUCTION}

The advent of antiretroviral therapy (ART) in the past two decades has significantly advanced the treatment and prevention of HIV worldwide. ${ }^{1}$ Rapid expansion of ART access to provide early treatment to all

\section{Strengths and limitations of this study}

- We constructed a dynamic-compartmental model that incorporates HIV risk status, HIV transmission and disease progression, and engagement in care to model HIV prevalence and incidence in India.

- We quantified the epidemiological benefits of achieving recent UNAIDS $90-90-90$ targets by 2020 in India and excess transmissions and mortality that would occur with delayed achievement.

- We are unable to provide financial implications of achieving such targets, as costs of healthcare strengthening (especially to improve retention in care) are unknown.

persons living with HIV (PLWH) has been identified as a key step in addressing the AIDS epidemic at a population level. ${ }^{2-6}$ As such, UNAIDS recently proposed the ambitious Fast-Track ('90-90-90') treatment targets, where, by $2020,90 \%$ of PLWH worldwide should know their diagnosis, $90 \%$ of these people should be on ART and $90 \%$ of these individuals (ie, $73 \%$ of all PLWH) should be virally suppressed, as a means to eliminate HIV as a public health threat by $20300^{7}$

Despite recent advances in HIV diagnosis and treatment, many low-income and middle-income countries face significant obstacles in reaching the 90-90-90 goals. ${ }^{8}$ In India, home to the third largest absolute burden of HIV worldwide, less than one-third of PLWH are currently virally suppressed, due to suboptimal diagnosis, treatment and retention in care. ${ }^{9-12}$ While implementing recent guidelines for early initiation of ART continue to remain a highpriority health intervention, other areas for improvement include increasing HIV awareness as well as decreasing the stigma and other sociocultural barriers that preclude many from seeking therapy and engaging in long-term care. ${ }^{13-17}$ 
The potential impact of achieving the 90-90-90 targets on the Indian HIV epidemic has yet to be described. We thus constructed a dynamic-transmission model to quantify new HIV infections and AIDS-related deaths over the next 15 years. In particular, we quantified the excess HIV transmissions and mortality that would occur if India delayed the healthcare strengthening necessary to reach these targets. Finally, we show that comprehensive healthcare strengthening in all aspects of HIV care is required to ultimately achieve the UN goal of eliminating HIV as a public health threat by 2030 .

\section{METHODS}

We used the Johns Hopkins HIV economic-epidemic model to project HIV prevalence, incidence, AIDS-related deaths and care continuum dynamics in India over a 15-year time horizon. Full details of the model are published elsewhere. ${ }^{18}{ }^{19}$ Briefly, our dynamic-compartmental model divides India's adult population (15-64 years) by sex, HIV infection status and HIV risk-profile (heterosexuals, men who have sex with men, people who inject drugs, female sex workers and high-risk men). HIV transmission is incorporated through sex (heterosexual and male homosexual) and needle sharing. HIV disease progression is dependent on viral suppression status. We also incorporated health system engagement by explicitly modelling rates of HIV screening and linkage to care, ART initiation, ART resistance, and disengagement and re-entry in care. Our model tracks usage of ART regimens, with switches made based on treatment failures and side effects (with rates of such events derived from published literature). We report on the percentage of PLWH on each of these regimens at various time points in our simulations. Model parameters, including HIV transmission probabilities and rates of engagement in care, were calibrated to reflect published epidemiological data on HIV prevalence, incidence and care continuum engagement in India between 2007 and 2011 (the last year for which there were complete epidemiological data). ${ }^{10} 2021$

In our base-case scenario, we implemented recent guidance recommending ART initiation irrespective of CD4 count. ${ }^{13}$ We then modelled a scenario in which the 90-90-90 targets would be achieved by 2020 . To determine the parameter set that would result in achievement of these targets, we iteratively increased model inputs influencing care continuum dynamics until each individual step along the care continuum matched desired outcomes (table 1). For example, to achieve the first 90 , we increased screening rates among the general population (testing every 5 years) and high-risk groups (annual testing) until $90 \%$ of PLWH were aware of their serostatus by 2020. We additionally projected epidemiological outcomes of the Indian HIV epidemic if achievement of the targets (as a whole) was delayed until 2025 and 2030. In these two scenarios, we assumed continuation of the base-case scenario until 2020 and 2025, respectively, at which point we incorporated the comprehensive healthcare strengthening required to reach 90-90-90 targets. We also considered a scenario where India continues a policy of deferred ART initiation at $\mathrm{CD} 4 \leq 350$ cells $/ \mathrm{mm}^{3}$ at current rates of care engagement (see the Results section). We conducted probabilistic uncertainty analysis by simultaneously varying all parameter values by Latin Hypercube sampling over specified ranges and report 95\% uncertainty ranges (URs). There are limited data describing the sensitivity ranges for the input parameters for our model. As such, the inputs for the sensitivity analysis were varied widely and largely based on the authors' experience from working in India, in conjunction with published literature.

\section{RESULTS}

Assuming adoption of recent guidance for early ART initiation (ie, initiation irrespective of $\mathrm{CD} 4$ count) in the current HIV care continuum, we project that 794000 incident HIV cases (95\% UR 571 000-1 104000) and 689000 AIDS-related deaths (95\% UR 468 000-976 000) would occur in India by 2030 (table 2). Implementation of early ART policies has minimal impact on achieving 90-90-90 targets at current rates of care engagement. At current rates of HIV screening, linkage and retention in care, we estimate that $72 \%$ of PLWH diagnosed with HIV would be on ART, and only $76 \%$ of PLWH on ART would be virally suppressed by 2030 .

Reaching UNAIDS 90-90-90 targets by 2020 would result in a drastic reduction of the HIV epidemic in India. If 90-90-90 were achieved, we estimate that 392000 new HIV infections (48\% reduction; 95\% UR $248000-559000$ ) and 414000 AIDS-related deaths (59\% reduction; 95\% UR $260000-598000$ ) could be averted by 2030 . Additionally, fewer than 20000 new HIV infections (95\% UR 12 000$30000)$ in 2030 would occur if India reached these targets on time. In this scenario of increased care engagement, we project that $26 \%$ of PLWH in India would require post-first-line regimens by 2020 , and more than half of HIV-infected individuals on ART would require secondline and third-line regimens by 2030 .

Delaying achievement of the Fast-Track targets resulted in markedly excess HIV transmission and mortality. With a delay of 5 years (ie, reaching targets by 2025 instead of 2020), India would incur an extra 211000 HIV infections and 201000 AIDS-related deaths by 2030 than if targets were reached on time. Furthermore, delaying fulfilment of 90-90-90 targets until 2030 (ie, delay of 10 years) resulted in marginal epidemiological benefits over 15 years compared to the base-case scenario (ie, implementation of early ART initiation alone). Specifically, with this additional delay, we project 709000 HIV transmissions (95\% UR 501000 $893000)$ and 617000 AIDS-related deaths (95\% UR $406000-789000)$ over 15 years; this translates to only $11 \%$ and $10 \%$ reductions in infections and deaths, respectively, over continuation of current care engagement (with early ART initiation). 
Table 1 Key model parameters

\begin{tabular}{|c|c|c|c|}
\hline Variable & Value & $\begin{array}{l}\text { Sensitivity } \\
\text { analysis }\end{array}$ & References \\
\hline \multicolumn{4}{|l|}{ HIV care continuum dynamics used in base-case scenario } \\
\hline $\begin{array}{l}\text { Percentage HIV testing in past } 12 \text { months among general } \\
\text { population }\end{array}$ & $3.2 \%$ & $1-7.5 \%$ & 22 \\
\hline Per cent HIV testing in past 12 months among high-risk groups & $17-31.8 \%$ & $5-60 \%$ & 22 \\
\hline $\begin{array}{l}\text { Percentage of newly diagnosed HIV patients with early linkage } \\
\text { to care* }\end{array}$ & $50-80 \%$ & $25-100 \%$ & 1023 \\
\hline Per cent of PLWH in care who become lost to follow-up yearly & $15-19.5 \%$ & $10-39 \%$ & 10112425 \\
\hline Median time spent lost to follow-up before return to care & 3 years & $2-4$ years & $\begin{array}{l}\text { assumption } \\
10112425\end{array}$ \\
\hline $\begin{array}{l}\text { Percentage of PLWH who develop resistance to first-line ART } \\
\text { after disengagement }\end{array}$ & $25 \%$ & $10-50 \%$ & $\begin{array}{l}\text { assumption } \\
26 \text {, assumption }\end{array}$ \\
\hline Per cent of PLWH on ART who develop resistance to ART & $7-10 \%$ yearly & $2-30 \%$ yearly & 112728 \\
\hline Median time after ART failure before treatment modification & $1.25-2$ years & $\begin{array}{l}8 \text { months- } \\
20 \text { years }\end{array}$ & 2728 \\
\hline $\begin{array}{l}\text { Median time after linkage to care before initiation of ART } \\
\text { (irrespective of CD4 count) } \dagger\end{array}$ & 3 months & $2-6$ months & 13 \\
\hline \multicolumn{4}{|l|}{ HIV care continuum dynamics used in $90-90-90$ scenario } \\
\hline $\begin{array}{l}\text { Per cent HIV testing in past } 12 \text { months among general } \\
\text { population }\end{array}$ & $20 \%$ & $15-25 \%$ & Calibrated \\
\hline Per cent HIV testing in past 12 months among high-risk groups & $\begin{array}{l}100 \% \\
\text { (ie, annual) }\end{array}$ & $80-100 \%$ & Calibrated \\
\hline Per cent of PLWH in care who become lost to follow-up yearly & $10-13 \%$ & $7.5-16.25 \%$ & Calibrated \\
\hline Median time spent lost to follow-up before return to care & 1 year & $8-15$ months & Calibrated \\
\hline Median time after ART failure before treatment modification & 4 months & $2-6$ months & Calibrated \\
\hline $\begin{array}{l}\text { Median time after linkage to care before initiation of ART } \\
\text { (irrespective of CD4 count) }\end{array}$ & 3 months & 2-6 months & Calibrated \\
\hline
\end{tabular}

\section{DISCUSSION}

Recent UNAIDS '90-90-90' treatment targets call for rapid scale-up of access and usage of ART over the next 5 years in an effort to eradicate the global HIV epidemic by $2030 .^{7}$ Our results show that achieving these targets on time-by 2020-can more than halve the epidemiological burden of HIV in India over 15 years, through substantial reductions in total incident cases and AIDS-related deaths. Fewer than 20000 HIV infections would occur in 2030 if these targets were reached in the next 5 years, an $84 \%$ reduction when compared to the 120000 infections that occur per year currently. ${ }^{31}$ Notably, our results suggest that even if achievement of the targets is delayed, the substantial reduction in yearly transmissions by 2030 would still occur-as long as the targets were eventually reached. Delaying fulfilment of the targets, however, would result in significantly excess total infections and mortality over 15 years, demonstrating the urgent need for comprehensive healthcare strengthening in India.

Despite updated guidelines for earlier ART initiation at higher CD4 counts, India currently continues a policy of deferred ART initiation at CD4 $\leq 350$ cells $/ \mathrm{mm}^{3}{ }^{3}{ }^{2} 2930$
Even in our base-case scenario, which assumes early ART initiation irrespective of CD4 count, our model projects that nearly half of PLWH aware of their serostatus will fail to achieve viral suppression by 2030-well short of current UNAIDS targets. Our modelling process demonstrates that despite its clear epidemiological benefits, earlier initiation of ART is necessary but not sufficient to achieve the Fast-Track targets by 2020. Our results are in contrast to previous studies suggesting that achieving 90-90-90 targets has a similar epidemiological impact as implementation of early ART initiation (at CD $4 \leq 500$ cells $/ \mathrm{mm}^{3}$ ) in sub-Saharan countries. ${ }^{32}$ This finding is likely explained by the fact that these countries, like India, experience significant attrition in HIV care that we explicitly accounted for in our modelling approach. ${ }^{33}$ Ultimately, early ART initiation addresses only one aspect of the care continuum, and without other improvements in care engagement (particularly early diagnosis, access to alternative regimens and longterm retention in care), its benefits are largely lost.

Current data suggest that a large portion of PLWH in India cannot access and gain the full benefits of earlier treatment due to infrequent HIV testing and late 
Table 2 Key model outputs

\begin{tabular}{|c|c|c|c|}
\hline & Incident cases & AIDS deaths & New HIV infections in 2030 \\
\hline Current care engagement (with & 794000 (95\% UR & 689000 (95\% UR & 46000 (95\% UR \\
\hline implementation of early ART guidance)* & $\begin{array}{l}571000-1104000) \\
\text { (reference) }\end{array}$ & $\begin{array}{l}468 \text { 000-976 000) } \\
\text { (reference) }\end{array}$ & $\begin{array}{l}29000-71000) \\
(62 \% \text { reduction from current } \\
\text { yearly incident cases }+)\end{array}$ \\
\hline \multirow[t]{3}{*}{$90-90-90$ by 2020} & 415000 (95\% UR & $280000(95 \%$ UR & 19000 (95\% UR \\
\hline & $289000-588000)$ & $194000-404000)$ & $12000-30000)$ \\
\hline & (48\% reduction) & (59\% reduction) & $\begin{array}{l}\text { (84\% reduction from current } \\
\text { yearly incident cases }+ \text { ) }\end{array}$ \\
\hline \multirow[t]{3}{*}{$90-90-90$ by $2025 \ddagger$} & 583000 (95\% UR & 488000 (95\% UR & $21000(95 \%$ UR \\
\hline & $479000-715000)$ & $379000-623000)$ & 14 000-31 000) \\
\hline & (27\% reduction) & (29\% reduction) & $\begin{array}{l}\text { (83\% reduction from current } \\
\text { yearly incident cases } \dagger \text { ) }\end{array}$ \\
\hline \multirow[t]{3}{*}{$90-90-90$ by $2030 \ddagger$} & 709000 (95\% UR & 617000 (95\% UR & $24000(95 \%$ UR \\
\hline & $501000-893$ 000) & 406 000-789 000) & 14 000-32 000) \\
\hline & (11\% reduction) & (10\% reduction) & $\begin{array}{l}\text { (80\% reduction from current } \\
\text { yearly incident cases } \dagger \text { ) }\end{array}$ \\
\hline \multicolumn{4}{|c|}{ 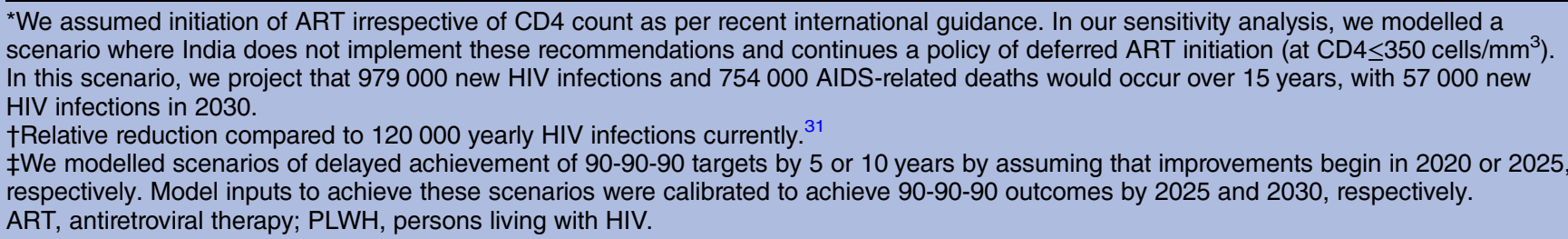 } \\
\hline
\end{tabular}

presentation to care. ${ }^{19} 20$ We found that with annual HIV screening for high-risk groups and testing every 5 years for the general population (a strategy shown to be cost-effective in India even as an isolated intervention), 9 out of 10 PLWH could achieve and maintain awareness of HIV status by 2020 and beyond. ${ }^{34}$ In the context of achieving 90-90-90 targets, improved screening would likely yield even greater epidemiological benefits and improved cost-effectiveness, as newly diagnosed individuals would rapidly integrate into care and achieve viral suppression. However, even after initiation of ART, many Indians face delays in detection of viral failure and subsequent treatment modification to alternative ART regimens, as only nine laboratories are qualified to cover viral load testing requests for all of India. ${ }^{9}$ As such, nearly half of PLWH in low-income Asian countriesincluding India-remain on failing regimens even 1 year after detection of treatment failure. ${ }^{27}$ By comparison, to achieve $73 \%$ viral suppression by 2020 , our model necessitated that PLWH were transitioned to second-line therapies on average within 4 months of viral failure. Moreover, one in five HIV-infected Indians in care becomes lost to follow-up, with only half eventually returning to care. ${ }^{1125}$ Achieving 90-90-90 targets in our model required a significant improvement over these care-engagement dynamics. Specifically, we were able to reach the target outcomes when $<10 \%$ of PLWH dropped out of care annually, with at least half returning to care by 1 year. Ultimately, our results highlight the need for comprehensive efforts to strengthen the HIV care continuum in India, in conjunction with immediate ART access for all PLWH irrespective of CD4 count.

While it has seen exponential growth in the past decade, India's ART programme faces numerous challenges in the coming years. Access to second-line ART is limited compared to first-line ART: of the three-quarter million Indians currently on ART, only 10000 are on a second-line regimen. ${ }^{9}$ Second-line ART regimens also cost up to six times more than first-line therapies. ${ }^{35}$ Furthermore, India's ART programme is currently facing widespread drug shortages due to recent bureaucratic changes and budget cuts, threatening to interrupt therapy for many. ${ }^{30}$ Our results suggest that an increasing proportion of PLWH will require postfirst-line regimens in the next 5 years if India reaches 90-90-90 targets, even with optimal engagement in HIV care minimising opportunities for viral failure. India must urgently set aside bureaucratic differences and restore full funding to its ART programme-with a particular emphasis on increasing second-line and third-line ART availability and affordability-if it is to reach the Fast-Track targets on time.

Our findings in India echo those of UNAIDS on the drastic effect of achieving 90-90-90 targets in the Asia/ Pacific region, though our projections of relative epidemiological impact are somewhat more modest when accounting for real-life gaps and cycles of care engagement. $^{7}$ Furthermore, in our base-case scenario, we assumed early ART initiation policies in India, as such guidelines are likely to be implemented in the coming years. Given the potentially optimistic assumption of this 
policy change, our model may actually underestimate the true epidemiological benefit of achieving the 90-90-90 targets. However, this difference in projections was reduced in our sensitivity analysis when we modelled a scenario where India continued a policy of deferred ART initiation.

Our model has several limitations. As there are a multitude of healthcare interventions that India can institute to reach the Fast-Track goals, the sets of parameters we defined in our various scenarios are likely not unique. However, we used the same iterative approach when arriving at the parameter sets for each scenario, thus minimising any inconsistencies. Our modelling approach does not take into account mid-course variations in the programmatic strategies for healthcare strengthening, as such growth patterns are likely nonlinear and thus not predictable. Additionally, we used published national estimates for HIV prevalence, incidence and care engagement to calibrate the model and generate outcomes for India as a whole; we are unable to comment on geographic heterogeneity given lack of complete data at a local and regional level. Finally, we were unable to provide the financial implications of reaching the 90-90-90 targets, as the costs for comprehensive healthcare strengthening (especially costs related to improved retention in care) are not known.

In conclusion, by achieving the UNAIDS Fast-Track targets on time, India can reduce the epidemiological burden of HIV by half and experience fewer than 20000 new HIV infections in 2030. This substantial reduction in HIV incidence by 2030 will likely occur even if India delays reaching the targets by 10 years, but at the cost of significantly excess overall transmissions and deaths. Comprehensive healthcare strengtheningwith earlier diagnosis and treatment, increased access to alternative ART regimens and improved long-term retention in care-is required if India is to reach the 90-90-90 targets by 2020 .

Contributors MVM and MS conceived and designed the study; MVM performed experiments with contributions from MS; MVM analysed and interpreted the data; MVM and MS wrote the manuscript with contributions from AG. All authors contributed to interpretation of results and critically reviewed and edited the manuscript.

Funding This project was supported with funds from the National Institute of Allergy and Infectious Diseases, National Institutes of Health, under grants K23Al089259, R01AI080417 and UM1AI069465; the Ujala Foundation; Gilead Foundation; and Wyncote Foundation. Funders had no role in the design or conduct of the study, analysis or interpretation of the results, manuscript writing or decision to publish results.

\section{Competing interests None declared.}

Provenance and peer review Not commissioned; externally peer reviewed.

Data sharing statement No additional data are available.

Open Access This is an Open Access article distributed in accordance with the Creative Commons Attribution Non Commercial (CC BY-NC 4.0) license, which permits others to distribute, remix, adapt, build upon this work noncommercially, and license their derivative works on different terms, provided the original work is properly cited and the use is non-commercial. See: http:// creativecommons.org/licenses/by-nc/4.0/

\section{REFERENCES}

1. Dieffenbach CW, Fauci AS. Thirty years of HIV and AIDS: future challenges and opportunities. Ann Intern Med 2011;154:766-71.

2. Cohen MS, Chen YQ, McCauley M, et al. Prevention of HIV-1 infection with early antiretroviral therapy. $N$ Engl J Med 2011;365:493-505.

3. Group ISS, Lundgren JD, Babiker AG, et al. Initiation of antiretroviral therapy in early asymptomatic HIV infection. N Engl J Med 2015;373:795-807.

4. Group TAS, Danel C, Moh R, et al. A trial of early antiretrovirals and isoniazid preventive therapy in Africa. $N$ Engl $J$ Med 2015;373:808-22

5. Smith K, Powers KA, Kashuba AD, et al. HIV-1 treatment as prevention: the good, the bad, and the challenges. Curr Opin HIV AIDS 2011;6:315-25.

6. Granich RM, Gilks CF, Dye C, et al. Universal voluntary HIV testing with immediate antiretroviral therapy as a strategy for elimination of HIV transmission: a mathematical model. Lancet 2009;373:48-57.

7. Joint United Nations Programme on HIV/AIDS. 90-90-90-an ambitious treatment target to help end the AIDS epidemic. Geneva: UNAIDS, 2014.

8. McMahon JH, Medland N. 90-90-90: how do we get there? Lancet HIV 2014;1:e10-11.

9. National AIDS Control Organization. NACO annual report 2014-15. India: NACO, 2015.

10. Alvarez-Uria G, Pakam R, Midde M, et al. Entry, retention, and virological suppression in an HIV cohort study in India: description of the cascade of care and implications for reducing HIV-related mortality in low- and middle-income countries. Interdiscip Perspect Infect Dis 2013;2013:384805.

11. Hamide A, Shamanna SB, Balaguru S, et al. Long-term outcome of HIV-infected patients treated at a tertiary care hospital in southern India. Natl Med J India 2014;27:134-7.

12. Mehta SH, Lucas GM, Solomon S, et al. HIV care continuum among men who have sex with men and persons who inject drugs in India: barriers to successful engagement. Clin Infect Dis 2015;61: $1732-41$.

13. World Health Organization. Guideline on when to start antiretroviral therapy and pre-exposure prophylaxis for HIV. Geneva: WHO, 2015.

14. Joglekar N, Paranjape R, Jain R, et al. Barriers to ART adherence \& follow ups among patients attending ART centres in Maharashtra, India. Indian J Med Res 2011;134:954-9.

15. Kumarasamy N, Safren SA, Raminani SR, et al. Barriers and facilitators to antiretroviral medication adherence among patients with HIV in Chennai, India: a qualitative study. AIDS Patient Care STDS 2005:19:526-37.

16. Geng EH, Nash D, Kambugu A, et al. Retention in care among HIV-infected patients in resource-limited settings: emerging insights and new directions. Curr HIV/AIDS Rep 2010;7:234-44.

17. Mishra S, Mountain E, Pickles M, et al. Exploring the population-level impact of antiretroviral treatment: the influence of baseline intervention context. AIDS 2014;28(Suppl 1):S61-72.

18. Shah M, Risher K, Berry SA, et al. The epidemiologic and economic impact of improving HIV testing, linkage, and retention in care in the United States. Clin Infect Dis 2016;62:220-9.

19. Maddali MV, Dowdy DW, Gupta A, et al. Economic and epidemiological impact of early antiretroviral therapy initiation in India. J Int AIDS Soc 2015;18:20217.

20. National AIDS Control Organization. NACO annual report 2013-14. India: NACO, 2014.

21. National AIDS Control Organization. NACO technical report: HIV estimates 2012. India: NACO, 2012.

22. UN Special Session of the General Assembly. Country progress report: India. Geneva: UNGASS, 2010.

23. Parchure R, Kulkarni V, Kulkarni S, et al. Pattern of linkage and retention in HIV care continuum among patients attending referral HIV care clinic in private sector in India. AIDS Care 2015;27:716-22.

24. Blutinger EJ, Solomon S, Srikrishnan AK, et al. Dropout from care among HIV-infected patients enrolled in care at a tertiary HIV care center in Chennai, India. AIDS Care 2014;26:1500-5.

25. Zhou J, Tanuma J, Chaiwarith R, et al. Loss to followup in HIV-infected patients from Asia-Pacific Region: results from TAHOD. AIDS Res Treat 2012;2012:375217.

26. Gupta A, Saple DG, Nadkarni G, et al. One-, two-, and three-class resistance among HIV-infected patients on antiretroviral therapy in private care clinics: Mumbai, India. AIDS Res Hum Retrovir 2010;26:25-31.

27. Zhou J, Li PC, Kumarasamy N, et al. Deferred modification of antiretroviral regimen following documented treatment failure in Asia: results from the TREAT Asia HIV Observational Database (TAHOD). HIV Med 2010;11:31-9. 
28. Boettiger DC, Kerr S, Ditangco R, et al. Trends in first-line antiretroviral therapy in Asia: results from the TREAT Asia HIV observational database. PLoS One 2014;9:e106525.

29. National AIDS Control Organization. Antiretroviral guidelines for HIV-infected adults and adolescents. India: NACO, 2013.

30. Sharma DC. Budget cuts threaten AIDS and tuberculosis control in India. Lancet 2015;386:942.

31. Joint United Nations Programme on HIV/AIDS. Gap Report 2014. Geneva: UNAIDS, 2014.

32. Granich R, Gupta S, Hersh B, et al. Trends in AIDS deaths, new infections and ART coverage in the top 30 countries with the highest AIDS mortality burden; 1990-2013. PLoS One 2015;10: e0131353.

33. Kranzer K, Govindasamy D, Ford N, et al. Quantifying and addressing losses along the continuum of care for people living with HIV infection in sub-Saharan Africa: a systematic review. J Int AIDS Soc 2012;15:17383.

34. Venkatesh KK, Becker JE, Kumarasamy N, et al. Clinical impact and cost-effectiveness of expanded voluntary HIV testing in India. PLoS One 2013;8:e64604.

35. National AIDS Control Organization. ART monograph: care, support, and treatment. India: NACO, 2011. 